\title{
Audiencia y servicio público en las televisiones autonómicas
}

institucional.us.es/ambitos/

\author{
Agustín Olmo López \\ Universidad de Sevilla \\ aolmo@us.es \\ José Antonio Navarro Moreno \\ Universidad de Sevilla \\ janava@us.es
}

English Version: Audience and public service in the regional televisions

Resumen

Las televisiones autonómicas, tan cuestionadas, nacieron para cubrir un espacio informativo inexistente hace tres décadas, y para potenciar un entretenimiento basado en elementos culturales más próximos. Su existencia ha sido importante para reflejar lo cercano, aunque ahora se intente diluir su labor con críticas dirigidas hacia su coste, su gestión o su independencia. El artículo busca demostrar que su aportación ha sido indudable también en la imagen identitaria de la Comunidad, haciendo un repaso cuantitativo a su seguimiento y su cumplimiento del servicio público, así como a su situación actual.

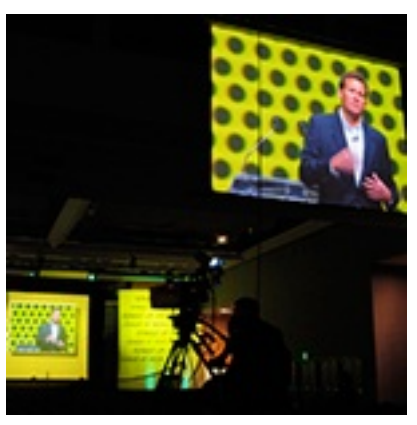

\section{Palabras clave}

Televisión, autonómica, identidad, servicio público.

Abstract

Regional televisions, nowadays as disputed, were born to meet a non-existent information space three decades ago, and for promoting an entertainment based on the closest cultural preferences. Theirs contribution to reflect

of the near items has been remarkable, although sometimes critics try to dilute theirs works with complaints

about theirs management or theirs independence. The article seeks to prove that theirs contribution were no doubt in the identity of the community, making a quantitative review of theirs follow-up and the compliance with the public service, and the actual situation.

\section{Keywords}

Television, regional, identity, public service.

\section{INTRODUCCIÓN}

La situación que ha vivido la televisión pública en general, y la autonómica en particular, durante los últimos tres años ha sido de auténtica zozobra. Cuestionadas desde un sector ideológico en la necesidad de su existencia y en su financiación, se vaticinaba una profunda transformación en la mayoría de ellas, dado que el gobierno abrió la posibilidad de introducir cambios profundos con una nueva ley que permitía su privatización o nuevas formas de gestión. Todo ello, además, se ha producido dentro de un panorama de crisis económica y de caída de audiencia, por lo que se han generalizado los comentarios negativos sobre la mayoría de ellas y se ha cuestionado su propia continuidad. Como consecuencia, en apenas unos meses se asistió a la adopción de medidas radicales en algunas de ellas, con recortes de presupuestos, despidos, privatizaciones, e incluso cierres. Eso derivó en un deterioro de su aceptación. 
públicos autonómicos (contemplando los segundos canales, canales internacionales, canales HD, etc.). Treinta años después de su nacimiento, contaban con una fuerte presencia que representaba cerca del $12 \%$ de cuota de pantalla y el 10\% de la inversión publicitaria en el sector de la televisión (según Kantar Media 2010), empleaba directamente a más de 6.000 profesional y entorno a ellas se crearon más de 650 productoras audiovisuales, con 12.959 trabajadores y una facturación de 206 millones de euros (FAPAE Memoria 2010). Se configuró así lo que según Richeri (2005) fue el sistema audiovisual regional más complejo de toda Europa, y en el que sólo 4 de las 17 comunidades autónomas no crearon su correspondiente televisión pública (Navarra, Cantabria, Castilla y León, y La Rioja), aunque sí surgieron privadas (tres de ellas son uniprovinciales lo que facilita su cobertura). Pero estos datos eran anteriores al inicio del desmantelamiento de una parte de este modelo. El sector ha cambiado notablemente con una profusión de canales y una renovación profunda del mercado audiovisual. La digitalización ha transformado el panorama en cuanto a señales, formatos, soportes y competencia, y todo ello ha coincidido con un momento convulso para su financiación.

\section{OBJETIVO}

Las televisiones autonómicas nacieron dentro de un contexto político que propugnaba la descentralización del Estado, y sirvieron para la construcción de un espacio autonómico de información que no existía.

En este artículo se pretende reflejar, desde un estudio real de los contenidos de las televisiones autonómicas, el grado de cumplimiento de la misión de servicio público que tienen encomendada, y que, en gran medida, era el argumento dado para justificar su existencia, y con ello ver hasta qué punto su funcionamiento es necesario en el panorama audiovisual español actual.

Igualmente, se analizará cómo se han visto afectadas por las medidas adoptadas en algunas Comunidades, disminuyendo su presencia e importancia como consecuencia de las mismas.

\section{METOLODOGÍA}

Tras un breve estudio teórico del marco de las televisiones autonómicas, bajo la óptica de su voz diferenciada y cercana orientada a resaltar los elementos identitarios, se analizarán los datos cuantitativos y estadísticos que ofrece su programación para comprobar hasta qué punto se acercan a sus objetivos fundacionales, así como para determinar cuáles son sus carencias. Igualmente, esos datos se compararán con los de las televisiones generalistas nacionales más destacadas para dibujar un panorama sobre su aportación específica en el ámbito comunicativo audiovisual. Para ello se han escogido cuatro años, con una cadencia de trianual, por estar marcados por lo que supuso el cambio tecnológico de la generalización de la televisión digital terrestre y, por tanto, de fragmentación del mercado. Se intenta ofrecer una visión general, aunque la captación de datos se ha efectuado dentro del mercado andaluz para poder comparar con una autonómica concreta, lo que condiciona que puedan darse matices exclusivos para esa comunidad autónoma.

\section{PANORAMA TELEVISIVO REGIONAL: 30 AÑOS DE TELEVISIONES AUTONÓMICAS}

Aunque las primeras experiencias en España se producen el año 1948, fue en 1956 cuando se iniciaron las primeras emisiones regulares. Entonces quedó establecido que era un servicio público estatal, cuyo objetivo era dar una información controlada, una difusión de la cultura interesada y unos contenidos generalistas.

En parte esta situación no difiere tanto del resto de radiotelevisiones públicas en Europa, y estaba ligada a la concepción de estado de bienestar que se estableció tras la posguerra mundial, y que asumía, según propone Bustamante (2008), que además de la protección en sanidad, tercera edad y desempleo, se debía garantizar el acceso a la educación, la cultura o la información, teniendo en ello un papel determinante las radiotelevisiones públicas. 
El hecho diferencial en España era la dictadura que gobernaba de manera centralista y uniforme, y eso reflejaba la televisión. Acabado el franquismo, se debía producir un tránsito hacia la democracia contemplando la realidad plural del país, lo que obligaba a adaptarse a lo que Bernat López reformuló con la definición francesa de "televisión de proximidad" [1].

Así se reconoció en 1980 en TVE, con la Ley 4/1980 de Estatuto de Radio y Televisión, que establecía la obligación de elaborar una programación específica en el ámbito territorial para cada nacionalidad o región. TVE disponía entonces de nueve centros fuera de la capital.

Si nos acogemos a la idea de Sánchez Noriega (2002: 26) de interpretar un "sistema mediático como un conjunto de comunicadores que, en la diversidad señalada tienen en común una presencia pública relevante y hasta hegemónica respecto a otras instancias sociales en orden a constituirse en "voz pública" y configurar el "pensamiento común", algunas autonomías entendieron que el servicio que daba TVE era insuficiente para cumplirlo. Por eso, sus gobiernos decidieron lanzar sus propios canales, creando un modelo insólito (Bustamante, 2006: 118). Así, la televisión vasca (ETB) empezó a emitir en 1982 para, entre otros objetivos, fomentar el euskera, y dado que ciertos Estatutos de Autonomía establecían como propias las competencias sobre los medios de comunicación social, se aprobó la Ley del Tercer Canal de Televisión que permitía la puesta en marcha de un nuevo canal en el ámbito de cada Comunidad. Inició su andadura la televisión catalana (TV3) en 1983 con el mismo propósito de normalizar el uso de la lengua autóctona, como hizo en 1985 la gallega (TVG). Las tres televisiones tenían como argumento principal la promoción de la lengua oficial diferente al castellano. Luego surgieronen otras comunidades, en las que imperó el criterio identitario-cultural, sin tener como justificación principal el uso de una lengua vernácula. En 1989 aparecen también las de Andalucía, Valencia y Madrid. Podría verse en ellas lo que señala Luhmann (1998) acerca de que los medios son autorreferentes y generadores de identidad que se alimenta del conjunto de sus producciones y se hace coherente con los valores dominantes en los que hay predominio de racionalidad mercantil. Construyen la realidad a través de la simplificación, la simulación o la falsedad apoyadas en la agenda que disuelve la memoria.

Con el tercer canal, esos gobiernos autonómicos se dotaban de una herramienta

con la quedifundir y promover cultura, tradiciones y lengua o hablas propias. Junto a ello, profundizar en la proximidad en lo relativo a la realidad informativa política, social y cultural sobre todo. Porque la noticia tiene como precepto ser alianza de las instituciones legitimadas ya que"el acto de producir la noticia es el acto de construir la realidad misma más que una imagen de la realidad"(Tuchman, 1983: 24). La audiencia, en principio refrenda esa oferta.

Por otro lado, la competencia crece vertiginosamente. En 1988 el gobierno aprobó la Ley de Televisión Privada y en 2005, con el Plan Técnico Nacional de TDT se concedieron nuevas licencias. En paralelo surgieron plataformas de pago, por satélite o cable, con multiplicidad de canales y señal de calidad digital, reguladas por la Ley de Telecomunicaciones de 1995. Igualmente, se incorporarán prestadores de servicio telefónico con paquetes televisivos a través de Internet (Telefónica con Imagenio, luego Fusión, u Orange con Orange TV).

Aumenta la competencia, y empieza a resentirse el seguimiento de la audiencia, pero se mantiene en buenos niveles de aceptación. Ante esa mayor oferta con las privadas y luego la TDT, se contrapone una búsqueda de especialización de contenidos, y los avances tecnológicos propiciaron el nacimiento de los segundos canales autonómicos como: Canal 33; ETB2, TVG2, Canal 2 Andalucía o Canal Nou 2, con programas para la infancia, culturales y deportivos.

Así, cuando se produjo el apagón analógico, en total en España había 109 canales digitales (concretamente en noviembre de 2010).

Con esa fragmentación disminuye la audiencia de cada canal, algo que se acentúa en los operadores autonómicos. Eso hace cuestionar, por parte de algunos partidos y de diversos medios, los costes de los mismos. Además, se han producido cambios en la forma de ver la televisión, y numerosos consumidores buscan en la web los programas, sin tener que someterse a los horarios de emisión. 
Incluso la red ha desbordado una de las nuevas realidades que señalaban Iracheta y Camuñas (2011: 267) que en el informe de la televisión en España publicado por la asociación de las televisiones comerciales (UTECA), apuntaban que ya no basta con entretener, informar o contar unos contenidos exclusivos o más interesantes que la competencia, sino que hay que llegar antes y mejor a cualquier usuario, y la red está creando circuitos paralelos a ello.

\section{SERVICIO PÚBLICO DE TELEVISIÓN}

En el Protocolo sobre el Sistema de Radiodifusión Pública de Ámsterdam, suscrito por los miembros de la Unión Europea en 1997, se afirma que "el sistema de radiodifusión pública está directamente relacionado con las necesidades democráticas, sociales y culturales de cada sociedad, y con la necesidad de preservar el pluralismo de los medios de comunicación". Se confirma en él, la importancia de los medios públicos. Un año más tarde, se publicó el Informe del Grupo de Alto Nivel de Política Audiovisual en el que concreta que la radiotelevisión pública ha de ser un medio fuerte, en competencia con las televisiones comerciales, y por tanto, con grandes audiencias.

En el caso de las autonómicas, cabría añadir que debe satisfacer, además, las demandas de información de la realidad territorial más próxima, proteger y reforzar las lenguas locales, y contribuir a la protección de los derechos sociales, económicos y culturales de las minorías.

Por todo ello, la televisión pública se presenta como una necesidad objetiva del conjunto de la sociedad, de los ciudadanos, razón por la que sus costes podrían considerarse como parte de un servicio social.

Si para las televisiones comerciales el objetivo principal es obtener los máximos beneficios; los canales públicos deben competir desde un criterio de complementariedad y deben combinar la oferta de programas ingeniosos que atraigan la participación de las masas con programas encaminados a satisfacer los gustos de audiencias minoritarias. Deben cubrir demandas de la audiencia que las televisiones comerciales no abordan, sin olvidar el entretenimiento.

El papel que reconoce el antropólogo Benedict Anderson (1993) a los medios de comunicación en la producción de un sentimiento de pertenencia a los habitantes de un mismo marco geográfico, se encuentra en casi todas las televisiones autonómicas que tienen como función la de vertebrar la identidad, la cultura de la región. Así lo recoge FORTA en su descripción del servicio público de las televisiones públicas autonómicas, de mayo 2011:

Emitir contenidos que velen y difundan la realidad de cada comunidad; contribuir a la difusión de las diferentes lenguas del Estado; construir un universo referencial propio donde las instituciones de cada comunidad reciban el tratamiento adecuado y preferente que tienen dentro de cada territorio; ser ventana de referencia de los grandes y pequeños eventos protagonistas de nuestro entorno, y contribuir a la difusión de las industrias culturales que nos rodean.

En la televisión, promover la identidad cultural, significa que se potencien los contenidos de la propia comunidad autónoma, y más cuando son exclusivos. De alguna manera sirven para reforzar lo que indica Carretero (2006: 108) ya que construyen el imaginario que cristaliza en mitos, fórmulas para superar la incertidumbre de la contingencia del mundo, creaciones individuales y colectivas más allá de lo racional, pues, de hecho, se apoyan a menudo en aspectos emocionales. Se trata de que lo simbólico y los imaginarios sociales se construyan y reproduzcan de forma comunicativa como realidad social (Berger y Luckman, 2001: 98)

Según los datos recabados por la consultora Accenture al elaborar un informe en 2012 para FORTA (basado en datos aportados por TVValencia, ARAGONTV, Castilla La Mancha TV, Canal Sur, TVGalicia, TV3-Cataluña, TeleMadrid y TPAsturias), la cantidad de contenidos centrados en la propia región que emitían las televisiones autonómicas, en 2010, representó un 8\% de su tiempo de programación, reflejado en 987 programas y 4.794 horas. En cuanto a los contenidos de divulgación de la cultura de la región y tradiciones regionales, las televisiones crean, subvencionan y promocionan eventos relacionados con dicha cultura, siendo el año 
señalado de 2.970 programas, traducido en 6.986 horas, lo que suponía aproximadamente un $11 \%$ de su tiempo de emisión. En el informe anual de 2010, Kantar Media adjudicaba a las TV autonómicas un 17\% de contenido cultural frente a un $5,6 \%$ de la televisión privada.

La actividad cultural de una región encuentra en su televisión autonómica una aliada para su promoción y difusión(efecto altavoz) y, a su vez, ésta dispone de una oportunidad para producir un contenido cultural de calidad y de arraigo.

Otra cuestión es la consideración de defensa de la lengua vernácula, pues en 2012 emitían 6 canales en catalán -tres de ellos generalistas-, 2 en euskera, 4 en gallego, 3 en valenciano, y 3 en balear.

Parte fundamental de la labor de las televisiones autonómicas es la promoción de la industria y el turismo de la comunidad autónoma. Este tipo decontenidos representaron durante el 2010 la emisión de 4.605 programas, 7.197 horas en total, lo que suponía aproximadamente un volumen del 12\% de la programación de dichos canales.

En contraste con las cadenas de ámbito estatal (privadas o públicas), la proximidad de los informativos autonómicos conseguía una mayor aportación a la audiencia. Según el Anuario TV 2010 de Kantar Media, los informativos supusieron, en promedio, una aportación del $29 \%$ a la audiencia de los primeros canales de las televisiones autonómicas públicas (un $24 \%$ si incluimos los segundos), frente a un $16 \%$ de la privada estatal. Destacaban TV3 con un $45,2 \%$, TVG con un $39,6 \%$ y TPA con un $38 \%$.

Las televisiones públicas autonómicas habían desarrollado, igualmente, otros formatos informativos diferentes que fomentaban la cohesión y el sentimiento de pertenencia, como eran 'Madrid Directo' y el resto de programas similares, o 'Pequeños, pero no invisibles', de AragónTV, o con el sentido de pertenencia aunque sea lejano, como 'Madrileños por el mundo', 'Afers exteriors' de TV3, etc.

Según los datos del informe de FORTA, en 2010 se emitieron un total de 6.568 programas que promocionaban la pertenencia a la comunidad, con un total de 27.219 horas de emisión, es decir, casi el $44 \%$ de su programación contribuía a la cohesión social y a la pertenencia al territorio.

También, en estos treinta años, las televisiones autonómicas han realizado una contribución indispensable al nacimiento de una industria de la imagen. Así, el $12 \%$ de sus programaciones era elaborado por estas empresas, cubriendo con 4.605 programas 7.197 horas de sus parrillas.

En definitiva, las televisiones públicas autonómicas se han consolidado como un instrumento potente y efectivo para fomentar el progreso social, económico y cultural de los territorios, y deben cumplir un papel como el que señalan Moragas y Prado (2001: 2) al decir que:

Las televisiones públicas, en un sistema competitivo y abierto, han de ser la garantía de un sistema de comunicación para todos, han de evitar la existencia de un sistema de voces limitado, han de ser el contrapeso del gran proceso de concentración que determina la nueva convergencia entre los sectores financieros, las telecomunicaciones y los mass media.

Observamos como existe una relación directa entre la comunicación regional y local y la aceptación de la audiencia, especialmente en el caso de los programas informativos, como aseguraba Navarro (1999: 57) "estos espacios informativos en los que se ofrecen noticias de carácter local y regional, han tenido siempre una gran aceptación por parte de la audiencia”.

Obviamente, también hay una objeción, y es que sobre el modelo audiovisual español siempre ha recaído la sospecha de su fuerte dependencia política. A pesar de los discursos oficiales y de las reformas habidas tanto en el ámbito estatal como autonómico, López Cepeda (2012: 419) no ve posible hablar de medios absolutamente independientes de los poderes políticos, debido a la gran influencia que ejerce en la opinión pública; aunque incluye que TVE, así como a las televisiones catalana y vasca, son las más que más pasos habían dado para ello. 


\section{ELEMENTO DE DISCUSIÓN: LAS PARRILLAS DE PROGRAMACIÓN Y AUDIENCIAS}

Al margen de esa cuantificación hecha en el punto anterior a partir de los datos facilitados por la propia FORTA con los que intenta resumir su servicio a las comunidades, hemos investigado considerando un intervalo de tres años completos para ver los programas de máxima audiencia de las principales cadenas autonómicas y comparar con los resultados de las cadenas nacionales y así poder establecer las diferencias significativas sobre cuáles son esos elementos diferenciales que caracterizan una televisión más próxima, y que redundan en la necesidad de su existencia. Los años escogidos han sido 2005 (el último completamente analógico, justo el anterior al inicio de la implantación de la TDT puesto que el plan diseñado por el gobierno empezaba en noviembre de ese año), 2008 (dos años después del comienzo de la TDT ya con una cobertura del $90 \%$ ), 2011 (el primero completo con TDT, puesto que el apagón analógico culminó en abril de 2010), y además 2014, el último año completo para comparar con datos recientes. En estos intervalos puede verse un progreso en los hábitos y consumos, con esa cadencia de tres años.

La base de información utilizada para ello proviene de la que procesa RTVA a partir de los datos suministrados por Kantar Media, empresa especializa en la medición de audiencias. Quiere esto decir, que el universo de población tomado es el de Andalucía para todas las televisiones nacionales; si bien, al referirse al resto de televisiones autonómicas no andaluzas, se considera su ámbito territorial.

\subsection{La televisión en 2005}

En 2005 hay doce televisiones autonómicas, contando entre ellas las cuatro que son segundas cadenas (Andalucía, Cataluña, Valencia y Euskadi tienen dos, y Madrid, Galicia, Castilla-La Mancha y Canarias una), y entre las nacionales están TVE con dos canales, y 4 privadas (justo ese año iniciaron sus emisiones Cuatro reconversión en abierto de Canal Plus- y La Sexta). Y es una de las privadas precisamente la cadena más vista en Andalucía, Antena 3 con un 21,6 \% (en el Estado un 21,3\%) seguida a 1,4 puntos por Canal Sur, y de Telecinco con 2,1 menos (sin embargo a nivel nacional es la primera con 22,3 \%). Televisión Española queda con un $16,5 \%$ de share $(19,6 \%$ estatal). Entre las autonómicas, la andaluza encabeza el ranking seguida de la catalana (TV3) con 19,6 \%; la vasca (ETB2) con 18,4 \% y la gallega (TVG) con un $17,2 \%$. La media de las autonómicas fue de un 17,3 de cuota de pantalla.

Hay un detalle a reseñar y es que, por franja de edad, en las públicas citadas el target de audiencia en el que triunfan es en todos los casos, salvo en ETB2, la de los mayores de 65 años, siendo el segundo el que va de los 65 a los 45 . En las privadas se observa que los espectadores son más jóvenes, pues Antena 3 triunfa entre los de 13 a 24 años, y Telecinco de 25 a 35 años. Curioso también es que en todas las públicas destaque su audiencia en las localidades de menos de 50.000 habitantes, (excepto en Valencia y Madrid), mientras que las privadas se imponen en las grandes ciudades.

Si nos fijamos en los 10 programas 10 más vistos del año, 7 son de una serie de humor de Antena 3 ('Aquí no hay quien viva') y tres a retransmisiones futbolísticas, (dos de la misma cadena anterior y otra de TVE). Todos superaron el $40 \%$ de share.

En todos los primeros canales de las autonómicas, salvo en Castilla-La Mancha que fue una película, encabezó la lista del programa más visto un partido de fútbol (variando según la comunidad). En los segundos canales hay dispersión: fútbol también, una carrera de fórmula 1, dibujos animados, y un programa de humor.

Si nos detenemos en el criterio de la aportación mensual en audiencia, en Canal Sur el programa que más ofreció fue 'Punto y Medio', un magazín conducido por Juan Imedio cuyo objetivo fundamental es dar compañía a la gente mayor (hay que señalar que aquí influye el número de horas de programación). Le sigue las 'Noticias' de mediodía, 'Andalucía Directo', 'Arrayán' (una teleserie andaluza) y cine. Es decir, dos espacios informativos, dos de entretenimiento y uno que se puede considerar que cumple una función social.

La 1 de Televisión Española encabezaba la lista con dos programas de cine, los dos magazines de mañana y tarde, y una telenovela. $Y$ en su segunda cadena contaba con uno deportivo, uno de cine y tres informativos (de reportajes de larga duración). 
Si observamos las privadas, Telecinco aupaba a los primeros lugares un talk show, un programa de prensa rosa, el magazine matutino de Ana Rosa Quintana, cine e informativos. Y Antena 3, situaba en primer y cuarto lugar el cine, luego en segundo y quinto sus informativos, y en medio 'Los Simpsons'.

\section{Cuatro y La Sexta eran irrelevantes para la audiencia.}

Del resto de autonómicas, destacar de TV3 que lidera su programación con un contenedor infantil, seguido de una teleserie ambientada en un barrio de Barcelona, el magazine matinal y luego dos espacios de cine. En su segunda cadena, hay tres programas infantiles, cine y un espacio deportivo. En Telemadrid, TVG, ETB2 y Televisión de Canarias (TVCAN) y Canal 9 (de Valencia) destaca el cine, siempre con al menos dos aportaciones entre las cinco primeras, y hay también siempre un informativo al menos.

Los informativos de las autonómicas (salvo en fin de semana), tanto en su primera como su segunda edición, son los más vistos. Destaca el espectacular 32,6 \% de share que consigue TV3 con sus noticias de mediodía.

Por géneros, coinciden privadas y públicas en aglutinar sus esfuerzos en los programas variados, pero las privadas apuestan más por la ficción y las públicas por los informativos. Ocupan el máximo de su programación con el género miscelánea Canal Sur, Antena 3, Telecinco y Cuatro con más de un $30 \%$ de su tiempo. En los de Ficción todas las demás autonómicas (con al menos un $36 \%$ ), salvo Telemadrid, que junto a TVE1, presenta un predominio de los Informativos (29 y 40,6\%, respectivamente). En todos los segundos canales se imponen los programas culturales con un 35,1\% de su tiempo el Canal Sur 2, y el 39,1 \% del canal catalán 33 .

\subsection{La televisión en 2008}

Este año ya se ha producido un notable incremento en la oferta televisiva. Hay 20 canales autonómicos. Destacan los tres de la televisión vasca; dos tienen Cataluña, Andalucía, Canarias, Asturias, Madrid y Valencia; y uno Galicia, Murcia, Castilla-La Mancha, Aragón e Islas Baleares. La media de share de todas ellas fue de un $14,08 \%$, superado por Canal Sur en dos puntos y 74 décimas. Eso sí, se aprecia una tendencia descendente debido a la fragmentación de la audiencia propiciado por la implantación de la TDT y la mayor oferta de canales, que aún así, apenas supone en ese momento un $4 \%$ de la cuota de mercado. También hay ya dos autonómicas privadas funcionando, por segundo año, Onda 6 en Madrid, y 8 TV en Cataluña, pero apenas consiguen un $0,6 \%$ de audiencia, eso sí, el doble que el año precedente.

Encabeza el ranking nacional Telecinco con un 18,1\% (15,2 \% en Andalucía) de la cuota de mercado, seguido a 2,1 puntos por Antena 3 (una décima más en Andalucía), y después de TVE1 con un 16,6 \% (11,8\% autonómico). Las otras privadas alcanzan un $8,5 \%$ La Sexta y un 7,6 \% Cuatro $(5,5 \%$ y $8,6 \%$ respectivamente en la región).

En general, cabe observar un progresivo retroceso de las cadenas autonómicas, al igual que en las televisiones nacionales existentes antes del 2005.

Canal Sur fue la dominadora entre la audiencia con un 16,82 \% de share, seguida con apenas 6 décimas menos por Antena 3, y casi un punto por Telecinco. TVE queda en el 14,37 \%. Las otras dos privadas no llegan al 8 porcentual. Se impone también la televisión andaluza al resto de cadenas autonómicas, seguida por las tres históricas, TVG con 15,49 \%, ETB2 con el 15,06 \% y TV3 con medio punto por debajo de la vasca. Esas cuatro son las únicas que superan la media de la FORTA (la federación de las autonómicas).

Canal Sur y los primeros canales de todas las públicas dominan la franja de los mayores de 65, excepto Asturias, Murcia y Euskadi donde esa franja es conseguida por su segunda cadena, la que emite en castellano. Las privadas, por el contrario se alzan con un público más joven, de 13 a 24 años Antena 3 y Cuatro, y de 25 a 44 Telecinco y La Sexta. Casi todas las segundas cadenas, menos La 2 de TVE y la de Asturias, tienen en el público más joven sus mayor cuota de seguimiento, considerando ETB1 también como segundo canal.

Se imponen las públicas en las localidades de menos de 50.000 habitantes, a excepción de Madrid y Canarias. Las privadas funcionan mejor en las poblaciones grandes (Antena 3 y La Sexta en las de más de medio millón y Telecinco y Cuatro en las intermedias). 
En cuanto a los 10 programas más vistos en Andalucía, 9 son partidos de fútbol 7 de Cuatro -retransmitió la Eurocopa en la que ganó España-, uno de Telecinco y otro de Antena 3) y el único no deportivo fue ocupado por TVE en sexto lugar con las votaciones del Festival de Eurovisión.

Los deportes pierden presencia en las autonómicas, -salvo en Baleares y Asturias con el fútbol, y ETB1 con fútbol y dos transmisiones de pelota vasca-. TV3 coloca entre dos partidos un programa de humor político ('Polònia'), y Aragón TV la inauguración de la Expo de Zaragoza antes de dos partidos de Copa del Rey. Canarias cuenta con un duelo regional como primera opción, seguido de un programa de humor y las campanadas de fin de año. Y en Valencia, entre dos capítulos de una serie de ficción local, figura un programa deportivo.

Pero hay varias en las que ninguno de esos acontecimientos figura entre las tres primeras opciones, como en Canal Sur donde lo más destacado en triple opción es un programa concurso sobre Copla; en Galicia con una teleserie llamada 'Padre Casares', o en Murcia donde triunfa el cine. También en Telemadrid y Castilla-La Mancha los dos primeros programas en aceptación son cine, y luego 'Madrileños por el Mundo', en un caso, y en el otro, un divulgativo aéreo de la geografía castellanomanchega.

En cuanto a los segundos canales, el deporte se alza con la hegemonía en 33, de Cataluña, y en dos puestos de La Otra de Madrid, quedando en medio uno de animales. En Canal Sur 2 todo lo más destacado es el concurso 'Cifras y Letras', y en Punt 2 de Valencia son distintos documentales, mientras que en la vasca ETB2 son dos informativos - uno especial y otro diario- y una gala; y en la segunda de Canarias son series de ficción.

Si nos fijamos en la aportación mensual a su audiencia en Canal Sur se impone 'La Tarde con María', un magazine, seguido de su informativo de mediodía y 'Andalucía Directo'; mientras en su segunda señal es un programa infantil, cine y uno juvenil.

En TVE1 en cambio es 'España Directo', 'Cine' y 'Amar en Tiempos Revueltos'; siendo en La 2 los grandes documentales, los Juegos Olímpicos de Pekín, y el Cine.

En Telecinco es un remedo de programa parecido al de las públicas que llevan el nombre de la región seguido de Directo, llamado ‘Está Pasando', seguido de ‘Escenas de Matrimonio' y el 'Programa de Ana Rosa'.

Antena 3 impone su informativo de las 3 como el más aceptado, y tras él, 'Los Simpsons' y el 'Cine de la tarde'.

En Cuatro y La Sexta no hay atisbo de informativos en los primeros lugares, con un concurso de baile y cine a diferentes horas en la primera; y fútbol, cine y una serie en la segunda.

En el resto de autonómicas se produce como norma general una combinación de cine y programas informativos en la acaparamiento de audiencias; siendo un espacio informativo el primero en Galicia, Valencia, Castilla-La Mancha, Baleares y Aragón.

Los informativos siguen siendo el punto fuerte de las públicas, y se imponen por lo general a todas las privadas. Los resultados son mejores en el de mediodía, con un sonoro 31,3\% del 'Telexornal 1' de TVG y el 24,7 \% de 'Telenoticies Migdia' de TV3 y el 23,5\% de 'Noticias 1' de Canal Sur. Sin embargo, los de TVE no superan a los de Antena 3.

Por géneros, de las nacionales más importantes, en tres privadas domina la ficción, y en la otra, en Telecinco, la miscelánea. En TVE1 los informativos superan el $47 \%$ de su tiempo, en La 2 los deportes y la cultura están casi el mismo tiempo, y entre ambos ocupan más de la mitad de la programación.

Entre las autonómicas hay variedad. Los informativos son los más programados en TV3 y Telemadrid; la miscelánea en Canal Sur, Punt 2 y ETB 1; la ficción se impone en Canal 9, TVG, ETB 2, Castilla-La Mancha, los dos canales canarios, Baleares, Aragón, Asturias y Murcia (es curioso este último caso pues supera la mitad de su tiempo total); los culturales son los más emitidos en Canal Sur 2 y 33; y los musicales son la base más importante de La Otra.

\subsection{La televisión en 2011}


Los datos de este año confirman el descenso de las públicas, inmersas, consecuencia además, de una merma en las subvenciones de sus gobiernos, y por tanto, de mayores dificultades financieras. Eso repercute en los resultados de una programación menos competitiva, ante un panorama de abundancia en la oferta.

El número de los canales autonómicos son 26 , debiéndose este aumento más a las segundas, terceras y hasta cuartas señales. 12 comunidades cuentan con su propio medio audiovisual: todas excepto La Rioja, Castilla y León, Navarra y Cantabria. Extremadura creó su cadena pero no se adscribió a la FORTA. Segundas cadenas cuentan 9 de las autonómicas (todas menos Islas Baleares, Murcia y Aragón). Una tercera ha sido puesta en marcha por Euskadi, Valencia y Cataluña. Y esta última, cuenta también con una cuarta.

Además, emitiendo en ámbito nacional hay 24 canales.

La audiencia media de las televisiones autonómicas públicas fue de 10,4\%, mientras que las autonómicas privadas quedaron por debajo de un punto $(0,8)$. Aparecen en los datos suministrados por Kantar Media 8 privadas autonómicas, tres en Cataluña, dos en Castilla y León, y una en Galicia, Madrid y Canarias, pero sus datos son apenas relevantes. Se aprecia también una incidencia de las televisiones de pago que cosecharon un $6 \%$ de la cuota de mercado.

Los dos primeros puestos de 2011 fueron en Andalucía para Telecinco con un 14,5\% y Antena 3 con un 12,8 \% (14,2 \% y 11,5 \% respectivamente en el ámbito nacional). Tras ellas TVE 12,5 \% (que sin embargo es la primera estatal con $14,5 \%$ ), y con menos de la mitad Cuatro (6,1\% en los dos niveles) y justo a un punto menos La Sexta a nivel nacional y a cuatro décimas en la autonomía. El resto de emisiones en TDT privadas conquista globalmente un $27,4 \%$ de share nacional y dos puntos menos en la región.

De las autonómicas, la más vista fue la catalana TV3 (14,1\%), luego la gallega TVG (12,3\%), y con siete décimas por encima de 10, igualan Aragón TV y Canal Sur. El resto queda por debajo de nueve puntos. De los segundos canales ninguno supera los dos puntos, y de los terceros el punto y medio.

En cuanto a los perfiles de edad, TVE se impone en los mayores de 65, pero esta vez le acompañan Telecinco, Intereconomía, y 13TV. Se acentúa esto en Canal Sur y TV3, y en ello coinciden todas las autonómicas en sus primeras y segundas señales. En Antena 3 son los mayores de entre 45 y 65 años. Mientras que los adolescentes y jóvenes se decantan por Cuatro y La Sexta, de las generalistas, y son también gran parte del target de las segundas y terceras marcas de las cadenas privadas (como Neox, FDF...). Hay también una serie de canales infantiles como Clan o Boing que domina el público de menos de 12 años.

Salvo las distintas señales de la Televisión Catalana, Telemadrid y Canal 9 (que lo hacen en las grandes ciudades de más de medio millón), todas las autonómicas se imponen en las poblaciones que no llegan a 50.000 habitantes. TVE en sus dos cadenas triunfa también en las grandes urbes, así como Antena 3 y La Sexta, mientras que Telecinco y Cuatro lo hacen en los núcleos medianos de población.

De los 10 programas más vistos del año, 9 fueron partidos de fútbol (6 de TVE, 1 de Canal Sur y dos de La Sexta), y uno, en sexto lugar, el 'Telediario Segunda Edición' de TVE del 3 de mayo, porque fue emitió durante el intermedio del partido de Champions League entre Real Madrid con el Barcelona, el segundo acontecimiento más visto del año.

Fue un año en que el fútbol arrasó en audiencias, siendo sólo los Telediarios situados entre los partidos los que se colocaron entre los 50 más vistos, junto con Eurovisión y 10 capítulos de 'Águila Roja', ambos de TVE. Coincide que ese año, los derechos de la Champions League los compartía TVE con las autonómicas, con lo que el predominio en las públicas fue total.

Por aportación a la audiencia, en Canal Sur destacan 'La Tarde Aquí y Ahora', -un magacín que fundamenta su éxito en dar compañía a gente mayor-, 'Andalucía Directo' -con información próxima-, y 'Arrayán', una teleserie ambientada en la Costa del Sol. En su segunda cadena son dos concursos, 'Cifras y Letras', y 'Taxi', junto a 'Cine'.

Los informativos siguen siendo los puntos fuertes de las autonómicas. Así, Canal Sur, TV3, TVG, Aragón TV y 
Asturias se imponen claramente a las nacionales al mediodía, superando el $20 \%, 31,3 \%$ del gallego 'Telexornal', el 28,9 \% del 'Telenoticies Migdia' catalán, y el 27,1\% de Aragón 'Noticias 1', y el 20,2 \% de TPA 'Noticias'. En la edición vespertina sólo la televisión catalana supera el 20 \% y supera claramente a la competencia, quedando la gallega muy igualada a las demás con un $14,2 \%$.

Por géneros, la información domina el tiempo de emisión en Canal Sur, TVE, TV3, TVG, Telemadrid, la 2 de Canarias y la asturiana. Telemadrid y TV3 superan el $40 \%$ de su tiempo destinado a espacios informativos.

La cultura prevalece en Canal Sur 2 y La 2, 33 (de Cataluña), Nou 2 (de Valencia) y ETB1. 33 roza el $80 \%$ de sus programas destinados a ese género.

La ficción se impone en Antena 3, Cuatro, La Sexta, Canal 9 (de Valencia), La Otra (de Madrid), Castilla-La Mancha, Canarias, Baleares, Aragón y Murcia. Un 45,7 \% de la Canaria es el guarismo mayor.

Por último, en Telecinco, los infoshow son los más programados, con un $28,5 \%$.

\subsection{Situación reciente. La televisión en 2014}

La profundización de la crisis económica y el aumento de los recortes han situado a todas las televisiones públicas en una difícil situación. RTVE rebajó su presupuesto en 2012 en doscientos millones de euros, otros 50 en el año 2013, que se mantuvieron en el 2014. Las televisiones autonómicas, a la reducción de la subvención, añadieron la caída estrepitosa de los ingresos publicitarios que en la mayoría ha significado una pérdida superior a los $2 / 3$ de lo que percibían seis años antes; en parte por al retraimiento económico, y en parte por el control de ese mercado que ejercen los dos grupos privados más poderosos que acaparan más del $80 \%$ de las inversiones.

La aprobación de la Ley 6/2012, de "modificación de la Ley General de la Comunicación Audiovisual" añadió más inestabilidad en la gestión de los servicios públicos de comunicación audiovisual autonómicos, pues permitía la gestión privada.

Ante eso, algunas han acometido medidas que reflejaban una predisposición ideológica de sus gobiernos respectivos, poco favorables a los medios públicos, como ha ocurrido con los entes de Madrid que ha ejecutado despidos cercanos al $75 \%$ de la plantilla (a 860 de 1.169 trabajadores), o el de Valencia, cuyo expediente de regulación de empleo afectaba a 1.198 personas de las 1.643 que conformaban la plantilla, y que acabó, finalmente, con el cierre definitivo. Entre ambas, acumulaban una deuda cercana a los 1.500 millones, y era recurrente presentarlas como el ejemplo más evidente de la gubernamentalización (Miguel y Casado, 2012: 130).

Otras corporaciones optaron por eliminar la segunda cadena, como en Castilla-La Mancha, Canarias, Andalucía, o Cataluña. En esta última, no sólo prescindió de una de ellas, sino que se fusionaron otras dos, anunciando posteriormente la extinción de más de 300 contratos. En Murcia, se entregó la gestión a un grupo privado, y unos meses antes se despidió a 230 de sus 260 trabajadores, llegando a no estar sin emitir durante unos meses. Igualmente, varias han decidido no continuar con su emisión por satélite.

Todo esto ha tenido su repercusión en la audiencia, pues todas las afectadas han visto disminuir su posición en el panorama audiovisual, con una caída importante en sus índices de seguimiento. Las que han aplicado medidas más traumáticas, se han resentido en mayor medida. Así, Telemadrid, Canal Nou y 7 Murcia, han tenido un descalabro mayor que las demás autonómicas, quedando notablemente por debajo de la media de la FORTA. Telemadrid consiguió un $4,2 \%$ en 2014, mientras que Canal Nou tuvo un 3,7 \% y 7 Murcia un $0,5 \%$ (los datos de estas dos últimas pertenecen a 2013 ya que acabaron 2014 sin emisión, y por tanto no se puede computar como año completo).

El share de todas las autonómicas públicas cayó del 10,4 \% en 2011 al 9,8 \% en 2012, en 2013 fue del 8,7 \% y 2014 lo cerraron con el 8,0 \%, según los datos de Kantar Media, empresa que recoge para las autonómicas públicas 18 señales: cuatro en Cataluña, tres en País Vasco, dos en Galicia, Madrid y Asturias, y una en Andalucía, Castilla La Mancha, Baleares, Canarias y Aragón. Autonómicas privadas había 6, dos en Cataluña y 
Castilla León, y una en Galicia y Madrid (Extremadura cuenta con un canal, pero no aparece integrado en el organismo de las televisiones autonómicas). Mientras que por satélite sólo había tres, la andaluza, la gallega y la vasca.

Aquí cabría establecer que esas renuncias a ocupar espacio público van en perjuicio de la salud democrática de una sociedad, pues según García Matilla (2003: 123124):

No hay servicio público cuando se da prioridad a los intereses coyunturales partidistas del grupo político en el Gobierno y éste se aprovecha del control y capacidad de influencia que tiene sobre el canal y sus profesionales [...] no se atiende al objetivo de servicio público cuando se da prioridad absoluta al interés por conseguir audiencia en detrimento de otras obligaciones de programación [...] se condena a un espacio a los peores lugares de programación sin tener en cuenta la audiencia a la que se destina [...] se dota de escasos recursos a los programas identificados como de servicio público [...] se utiliza este tipo de programa como forma de castigo a los profesionales [...] se utilizan los conceptos de «educativo» o «cultural» como cajón de sastre en el que incluir espacios sin destinatarios definidos [...] se elude dar acceso al medio a grupos sociales representativos.

Estaríamos asistiendo a un empobrecimiento en la

\section{Tabla 1. Datos audiencia 2014}

\section{AUTONÓMICAS PÚBLICAS}

\begin{tabular}{|c|c|c|c|c|}
\hline & & RANKING & SHARE 2014 & SHARE 2013 \\
\hline 1 & $s$ & -3 & 12,6 & 13,5 \\
\hline 2 & 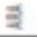 & $\approx$ & 11,3 & 11,5 \\
\hline 3 & $\exists$ & $\subset$ & 10,0 & 10,9 \\
\hline 4 & 迹 & 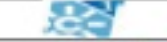 & 9,5 & 9,9 \\
\hline 5 & $\mathrm{~g}$ & $\Rightarrow 2$ & 9,0 & 9,6 \\
\hline$\epsilon$ & 吾 & {$[<-8]$} & 7,3 & 7,2 \\
\hline 7 & 5 & $=17$ & 6,2 & 5,4 \\
\hline a & $\Xi$ & (B) & 5,8 & 5,9 \\
\hline 9 & 즐 & T) & 4,6 & 4,4 \\
\hline 이 & 3 & $\Leftrightarrow$ & 4,2 & 3,8 \\
\hline 1 & $\mathrm{~s}$ & $\Rightarrow 0$ & 2,0 & 2,1 \\
\hline 2 & $\overline{3}$ & $\Leftrightarrow 3$ & 1,6 & 1,7 \\
\hline$=$ & इ & $x=4$ & 1,3 & 1,2 \\
\hline 4 & $\overline{3}$ & 23 & 1,2 & 1,4 \\
\hline 5 & 욜 & $\Rightarrow 3$ & 0,9 & 0,9 \\
\hline$\varepsilon$ & 3 & $\mathrm{Cl}_{2}$ & 0,8 & 0,9 \\
\hline & 3 & 5:- & 0,8 & 0,7 \\
\hline a & 5 & 료 & 0,7 & 0,7 \\
\hline
\end{tabular}

Fuente: Kantar Media

pluralidad, ya que como señalaba sobre este servicio

público en un sentido amplio Emilio Lledó en el preámbulo del informe del grupo de expertos para la reforma de la Televisión encargado por el gobierno de Rodríguez Zapatero, es "un bien social que, en cierto sentido, permite fomentar los indudables beneficios de la maquinaria que sustenta la sociedad de la información".

No en vano, las televisiones comerciales tienen como objetivo captar más audiencia y conseguir más beneficios. Privadas empezaron emitiendo en ámbito estatal 26, y acabaron 20, dado que en cumplimiento de una sentencia del Tribunal Supremo hubo un apagón en 6 de ellas.

Las televisiones de pago que cosecharon un 6,2 \% de la cuota de mercado. La caída de las públicas es notoria, siendo el primer puesto para Telecinco con un 14,5\% y Antena 3 con un 13,6 \% (en Andalucía un 13,9 y 13,7 respectivamente). Tras ellas TVE $10 \%$ (punto y medio menos en la región), Cuatro $(6,6 \%$ nacional y una décima más en Andalucía) y La Sexta (6,2 \% estatal y un punto más en la autonomía). Las emisiones en TDT privadas conquistan un $27,4 \%$ de share.

De entre las autonómicas, las más vistas fueron TV3 (12,6\%), Aragón TV (11,3\%) y TVG (10 \%), y a medio punto Canal Sur. Ninguno de los segundos canales supera los dos puntos.

Por perfiles de edad, claramente las televisiones públicas (Canal Sur, resto de primeros canales autonómicos y TVE) el sector de público que domina es el de los mayores de 65, mientras que Antena 3 capta es la más aceptada en la franja de los 25 a los 64, Telecinco en el de 13 a 24 años, mientras que en Cuatro y La Sexta su público está más repartido en las franjas intermedias. El tramo infantil es para Boing.

Salvo la televisión catalana y Telemadrid (por el peso de Barcelona y Madrid), todas las primeras cadenas autonómicas se imponen en las poblaciones que no llegan a los 50.000 habitantes; TVE en las localidades intermedias que no superan el medio millón, al igual que ocurre con Telecinco. El resto de la generalistas privadas tiene en las grandes urbes su mayor cuota de público.

Todos los 10 programas más vistos del año fueron partidos de fútbol, y tuvieron que ver con la celebración del 
Mundial de este deporte, excepto el del final de la Copa del Rey entre Real Madrid y Barcelona.

El deporte rey acaparó audiencias, pues entre los treinta primeros programas sólo se encuentra un Telediario y el concurso La Voz Kids.

Por aportación a la audiencia, en Canal Sur destaca 'La Tarde Aquí y Ahora', 'Andalucía Directo', 'Noticias 1'y 'Noticias 2', y de manera excepcional "Yo también canté el villancico de Canal Sur". Ya no se contempla segunda cadena, pues para reducir costes la programación es la misma que en la primera, pero con traducción a la lengua de signos.

Los informativos siguen siendo los puntos fuertes de las autonómicas y se imponen a las nacionales al mediodía, superando el $25 \%$ las de Aragón, Galicia y Cataluña. En la edición vespertina las mismas pasan del $20 \%$.

\section{CONCLUSIONES}

Si las televisiones autonómicas ocuparon en sus orígenes un espacio de ilusión para sus potenciales espectadores, y contaban con una misión definida por sus gobiernos responsables en busca de reforzar identidades, el paso del tiempo parece haber diluido ese apoyo. En esa trayectoria, su importancia es proporcional al respaldo financiero que les ha otorgado el gobierno autonómico del que dependen. Y, si esa dependencia económica fue en sus inicios positiva, a la larga, no se vieron dotadas de elementos que les permitieran conducirse de una manera estable.

Es un modelo televisivo que se ha creado en paralelo al modelo político autonómico, porque en casi ninguna comunidad existían referentes informativos de esa dimensión, o con esa vocación. Su misión era aportar vertebración, identidad, $u$ ofrecer espacios de pluralidad dentro de su ámbito, y esto lo han intentado cumplir. Ahora bien, es difícil generalizar ante un espectro tan variado como son esas televisiones. Si están en cuestión ahora es porque se cuestiona el propio estado autonómico desde ciertos sectores políticos y mediáticos, y porque las emisoras privadas lanzan mensajes de su oneroso mantenimiento, ya que no quieren esa competencia en el mercado publicitario.

Todas empezaron imitando el esquema de empresa que tenía RTVE, aunque con el tiempo ha ido adaptándose a otras dinámicas.

La proliferación de canales privados, en lugar de proporcionarles un lugar como alternativa de servicio público cercano, parece más bien haber diluido su presencia. Su propio crecimiento quizás se ha desbordado en los últimos años.

Como Ramonet (2002: 8) señalaba, la transformación digital de la televisión está haciendo desaparecer el concepto de "público en general", y esto debilita la función de cohesión nacional que tenía el medio. Así, con el avance tecnológico aparecen nuevos retos, que son resultado de apuestas económicas muy cuantiosas, de apuestas políticas y de retos culturales no menos importantes. Ante ellos, quizás sean las televisiones autonómicas, junto a las locales, la primera línea de combate en ese proceso que él aventura producirá unas audiencias segmentadas, por grupos y de pago.

Las televisiones autonómicas sí que han centrado una parte de su acción en ser garantes de difundir la especificidad de sus zonas, donde han encontrado sus nichos de audiencia, pero los target de sus seguidores no son los más atractivos. Son en general, los mayores de 65 años, y residentes en poblaciones pequeñas.

Su dimensión depende en parte de la descentralización a la que se han sometido la mayoría, al estar presentes en todas sus provincias.

Los datos ofrecen una importante dedicación a su actualidad, su cultura, y su representación institucional. Sin duda, las menos cuestionadas tienen en común el ser las tres primeras, y coinciden en ser consideradas de comunidades históricas, y si bien el idioma es su hecho diferencial, no es la única causa, puesto que en la 
Existe una relación directa entre las televisiones que más audiencia han perdido y la actitud de sus gobiernos ante las mismas, pues en aquellas comunidades donde los gobiernos (todos del Partido Popular) han manifestado su falta de interés por una televisión pública autonómica, y su voluntad de cambiar la gestión, la audiencia ha sufrido un descalabro mayor que en el resto de las televisiones.

El valor mayor de su servicio público suele recaer en los informativos, que gracias al factor proximidad resultan atractivos. No obstante, este punto es más destacado a mediodía que por la noche. También otro tipo de programas informativos, como los de media tarde que recogían una parte en Directo, han sido relevantes en la apreciación de las cadenas.

Los mayores éxitos de los programas no dedicados a las noticias se solían centrar en elementos particulares de sus regiones, como teleseries ambientadas en sus entornos ('Arrayán' o 'Padre Casares'), o por tener elementos culturales cercanos ('Se llama Copla' o 'Se escribe con Jota'), o por un humor sobre sus especificidades ('Polònia' o 'Vaya Semanita'), sin olvidar la búsqueda de las raíces ('Pequeños pero no invisibles' o 'Tal como somos').

Gracias a ellas se ha desarrollado un importante sector industrial que ahora está en peligro. E igualmente, han hecho una aportación incontestable al patrimonio, al crear archivos audiovisuales de un valor incalculable.

\section{REFERENCIAS BIBLIOGRÁFICAS}

\section{Libros}

ANDERSON, B. (1993): Comunidades Imaginadas. Buenos Aires: Fondo de Cultura Económica,

ARTERO, J. P. et alia (2011): La televisión en España. Informe 2011. Madrid: Ediciones IESE

BERGER, P. L. y LUCKMAN, T. (2001): La construcción de la realidad social. Buenos Aires: Amorrortu editores.

BUSTAMANTE, E. (2006): Radio y televisión en España. Historia de una asignatura pendiente de la democracia. Barcelona: Gedisa.

CAMPO VIDAL, M. et alia (2010): La industria audiovisual en España. Escenarios de un futuro digital. Madrid: Academia TV y EOI.

DE MORAGAS, M., GARITAONAENDÍA, C. y LÓPEZ, B. (1999): Televisión de proximidad en Europa. Experiencias de descentralización en la Era Digital. Barcelona: Aldea Global.

GARCÍA MATILLA, A. (2003): Una televisión para la Educación. La utopía posible. Barcelona: Gedisa.

LUHMANN, N. (1998): Complejidad o modernidad: de la unidad a la diferencia. Madrid: Editorial Trotta.

NAVARRO MORENO, J.A. (1999): La televisión local. Andalucía, la nueva comunicación. Madrid: Fragua

MIGUEL DE BUSTOS, J. C. y CASADO DEL RIO, M. A. (2012): Televisiones autonómicas. Evolución y crisis del modelo público de proximidad. Barcelona: Gedisa.

RAMONET, I.: "La gran mutación”. En RAMONET, I. (ed.) (2002): La post-televisión. Multimedia, Internet y globalización económica. Barcelona: Icaria, pp.7-15

RICHERI, G. (2005): Le televisión pubbliche nelle regioni d'Europa. Roma: RAI.

SÁNCHEZ NORIEGA, J. L. (2002): Crítica de la seducción mediática. Comunicación y cultura de masas en la 
opulencia informativa. Madrid: Tecnos.

TUCHMAN, G. (1983): La producción de la noticia. Estudio sobre la construcción de la realidad. Barcelona: Gustavo Gili.

\section{Artículos en revistas}

BUSTAMANTE, E. (2008): "Amenazas y posibilidades del sistema audiovisual europeo en la era digital". Cuadernos de Información, Vol. 2, n 23, (pp. 82-95), Santiago, Chile. [Fecha de consulta: 24 de mayo de 2015]. http://cuadernos.uc.cl/uc/index.php/CDl/article/view/98.

CARRETERO PASÍN, A. E. (2006): "La persistencia del mito y de lo imaginario en la cultura contemporánea". Política y Sociedad, Vol. 43, $n^{\circ} 2$ (pp. 107-126), Madrid. [Fecha de consulta: 19 de abril de 2015]. http://revistas.ucm.es/index.php/POSO/article/view/23692.

DE MORAGAS, M. y PRADO, E. (2001): "Repensar la televisión pública en el contexto digital”, en Portal de la Comunicación, Cerdanyola del Vallès (Barcelona). [Fecha de consulta: 10 de mayo de 2015]. http://www.portalcomunicacion.com/both/opc/tvp.pdf

LÓPEZ CEPEDA, A. M. (2012): "Modelos audiovisuales públicos en España. Perfil profesional, empresarial y político de sus principales órganos internos de gestión”. Comunicación y Sociedad, Vol. 25, n 1, (pp. 399-427), Pamplona (Navarra). [Fecha de consulta: 4 de junio de 2015]. http://www.unav.es/fcom/comunicacionysociedad/es/articulo.php?art_id=418.

[1] Término utilizado por primera vez en Francia para nombrar las desconexiones de France3.

Ámbitos. Revista Internacional de Comunicación, n.29, año 2015, tercer trimestre (verano). Recibido: 02/04/2015

Aprobado: 05/06/2015 